RADIOLOGY-ORIGINAL ARTICLE

\title{
Reference absolute and indexed values for left and right ventricular volume, function and mass from cardiac computed tomography
}

\author{
Jadranka Stojanovska, Hutsaya Prasitdumrong, Smita Patel, Baskaran Sundaram, Barry H Gross,
} Zeynep N Yilmaz and Ella A Kazerooni

Division of Cardiothoracic Radiology, Department of Radiology, University of Michigan Health System, Ann Arbor, Michigan, USA

J Stojanovska MD, MS; H Prasitdumrong MD; S Patel MBBS, MRCP, FRCR; B Sundaram MBBS, MRCP, FRCR; BH Gross MD; ZN Yilmaz MD; EA Kazerooni MD, MS.

\author{
Correspondence \\ Dr Jadranka Stojanovska, Department of \\ Radiology, University of Michigan Health \\ System, UH B1-132 Taubman/Box 0302, 1500 \\ East Medical Center Drive, Ann Arbor, MI \\ 48109-0302, USA. \\ Email: jstoanov@umich.edu
}

Conflict of interest: This work has not been funded by any funding agency.

Submitted 30 December 2013; accepted 5 April 2014.

doi:10.1111/1754-9485.12186

\begin{abstract}
Introduction: Left ventricular (LV) and right ventricular (RV) volumetric and functional parameters are important biomarkers for morbidity and mortality in patients with heart failure.

Purpose: To retrospectively determine reference mean values of LV and RV volume, function and mass normalised by age, gender and body surface area (BSA) from retrospectively electrocardiographically gated 64-slice cardiac computed tomography (CCT) by using automated analysis software in healthy adults.

Materials and Methods: The study was approved by the institutional review board with a waiver of informed consent. Seventy-four healthy subjects (49\% female, mean age $49.6 \pm 11$ ) free of hypertension and hypercholesterolaemia with a normal CCT formed the study population. Analyses of LV and RV volume (end-diastolic, end-systolic and stroke volumes), function (ejection fraction), LV mass and inter-rater reproducibility were performed with commercially available analysis software capable of automated contour detection. General linear model analysis was performed to assess statistical significance by age group after adjustment for gender and BSA. Bland-Altman analysis assessed the inter-rater agreement.

Results: The reference range for LV and RV volume, function, and LV mass was normalised to age, gender and BSA. Statistically significant differences were noted between genders in both LV mass and RV volume $(P$-value $<0.0001)$. Age, in concert with gender, was associated with significant differences in RV end-diastolic volume and LV ejection fraction ( $P$-values 0.027 and 0.03 ). Bland-Altman analysis showed acceptable limits of agreement $( \pm 1.5 \%$ for ejection fraction) without systematic error.

Conclusion: LV and RV volume, function and mass normalised to age, gender and BSA can be reported from CCT datasets, providing additional information important for patient management.
\end{abstract}

Key words: cardiac $\mathrm{CT}$; function; left ventricle; mass; right ventricle; volume.

\section{Introduction}

Left ventricular (LV) and right ventricular (RV) volumetric and functional parameters are important biomarkers for morbidity and mortality in patients with heart failure (HF). Knowledge of these parameters relative to mean reference values and accurate and reliable determination of these values are crucial for prognosis and treatment in patients with HF. ${ }^{1-3}$
The assessment of LV and RV volumes, function and mass can be performed non-invasively using echocardiography, electrocardiographic (ECG)-gated multidetector cardiac computed tomography (CCT) or cardiac magnetic resonance (CMR) imaging, or invasively with cardiac catheterisation. Two-dimensional (2D) echocardiography $(\mathrm{ECHO})$-derived volumes may not represent true chamber volumes when compared to CCT or CMR, both of which demonstrate cardiac chamber anatomy 
in an omnidimensional fashion ${ }^{4}$ due to the complex geometry of cardiac chambers and the presence of trabeculations, which pose significant challenges. Threedimensional ECHO should be more comparable to the reference standard of CMR than $2 \mathrm{D} \mathrm{ECHO},{ }^{5}$ but CMR is considered to be the current non-invasive reference standard for evaluation of cardiac anatomy, volume and function. ${ }^{6}$ However, CMR may not be feasible for reasons such as claustrophobia, unavailability and presence of pacemakers/defibrillators, which are relative contraindications for $M R$ imaging. In this group of patients, CCT may be a valid option.

CCT has good spatial and temporal resolution, and when performed using retrospective ECG gating, with data acquisition throughout the entire cardiac cycle, it can optimally anatomically orient the heart for the assessment of the cardiac chamber anatomy, volume, ejection fraction and cardiac mass, with no additional contrast administration. ${ }^{7}$ CCT has excellent correlation with CMR, generally regarded as a current standard of reference for analysis of left ventricular function.

LV and RV volumes vary by patient age, gender and body surface area (BSA). ${ }^{8,9}$ Significant differences in the cardiac chamber reference values may exist when using different sequences with MRI and even when using different software analysis packages. ${ }^{10,11}$ Therefore, modality and software analysis require specific mean reference values for LV and RV function, volume and mass. The purpose of this study is to establish the CCT-based reference mean values (absolute and indexed) for LV and RV volumes, function and mass, normalised by age, gender and BSA, in healthy adults free of hypertension and hypercholesterolaemia by means of automated analysis software with minimal manual correction by the user.

\section{Materials and methods}

\section{Study design}

A retrospective cohort study of ECG-gated multidetector cardiac CT scans performed at our institution was used to establish mean reference values of LV and RV volume and function indexed to age, gender and BSA using automated analysis software package in healthy adults free of hypertension and hypercholesterolaemia by means of descriptive statistics and general linear model analysis.

\section{Study population}

The medical records and CCT reports of 675 adult subjects with atypical chest pain presenting either in the emergency department (ED) or in an outpatient clinic who underwent CCT from 2006 to 2011 were retrospectively reviewed (Fig. 1). Seventy-four subjects (36 females and 38 males, mean age $49.6 \pm 11$ years, age range 31-72 years) who fulfilled the inclusion criteria of normal cardiovascular function with no evidence of coronary artery disease (normal ECG and normal retrospectively gated CCT angiogram (CCTA) of the coronary arteries) and had no risk factors for coronary artery disease such as hypertension, hypercholesterolaemia, diabetes or structural heart disease (normal history, physical examination and normal echocardiography were available and confirmed in 47 patients). No major adverse cardiac events were noted at subsequent 6 -month chart review in any patient. All subjects had a low pretest probability for coronary artery disease based on Framingham criteria. ${ }^{12}$ ED patients were discharged with a diagnosis of non-cardiac chest pain, such as pain
Review of medical charts of adult subjects $(N=675)$ with atypical chest pain from 2006 to 2010

\section{Normal subjects $\geq 18$ years $(N=74)$ free of CAD, HTN and hyperlipidaemia}

CT suite:

Vitals (HR, BP), weight, height, $\mathrm{Cr}$, pregnancy test

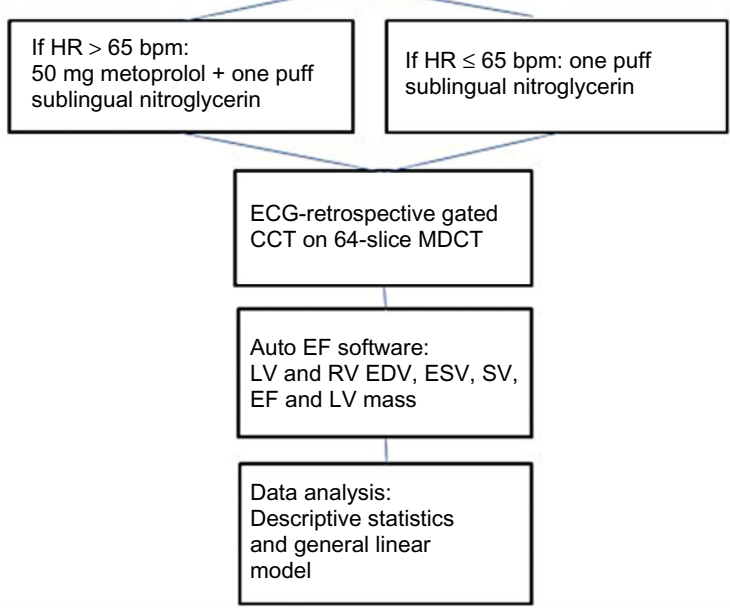

Fig. 1. Study design flow. CAD, coronary artery disease; CCT, cardiac computed tomography; $\mathrm{Cr}$, creatinine; EDV, end-diastolic volume; EF, ejection fraction; ESV, end-systolic volume; HR, heart rate; HTN, hypertension; LV, left ventricle; MDCT, multidetector computed tomography; RV, right ventricle; SV, stroke volume. 
of musculoskeletal or gastrointestinal origin or panic attack. Height, weight, blood pressure and heart rate during the CCTA examination were recorded for all subjects. BSA was calculated using the Mosteller formula. ${ }^{13}$ Four age groups by decade were established: group 1 = ages 30-39, group 2 = ages 40-49, group $3=$ ages 50-59 and group $4=$ ages $60-70$. The study was approved by the institutional review board with a waiver of informed consent.

\section{ECG-gated CCT technique}

All subjects underwent assessment of vital signs (blood pressure and heart rate) at least an hour before CCTA. Only subjects with heart rate higher than 65 beats per minute (bpm) underwent oral premedication with $50 \mathrm{mg}$ of metoprolol at least $45 \mathrm{~min}$ before the scanning. A heart rate of $\leq 60 \mathrm{bpm}$ was achieved in all patients during the scan. All subjects underwent premedication with one puff of sublingual nitroglycerin 1-5 min before CT scanning.

All scans were acquired in the supine position with the patient's arms elevated above and behind the head on a 64-row multidetector CT (MDCT; Lightspeed VCT, GE Healthcare, Milwaukee, WI, USA). After the localising scan was obtained, image acquisition was performed in the craniocaudal direction at end-inspiration within a single breath hold, while the patient's ECG trace was recorded simultaneously. Scan z-axis coverage ranged from $2 \mathrm{~cm}$ above the most cephalad coronary artery to $2 \mathrm{~cm}$ below the cardiac apex. Scan parameters were the following: slice thickness, $0.625 \mathrm{~mm}$; tube voltage, $100-$ $120 \mathrm{kVp}$; mA, adjusted for patient size based on a body mass index (BMI) look-up table; and tube rotation, $0.35 \mathrm{~s}$.

Iso-osmolar contrast material (Visipaque 370, GE Healthcare) was administered through an 18-gauge intravenous canula placed in the right antecubital fossa. A test bolus of $15 \mathrm{~mL}$ of contrast material was injected at $5 \mathrm{~mL} / \mathrm{sec}$ with the region of interest in the aortic root at the level of the left main coronary artery. For each patient a Hounsfield unit time graph was obtained from which the scan delay was calculated as peak enhancement plus $6 \mathrm{~s}$. The dedicated CCTA acquisition was then acquired using a triphasic contrast bolus with a total of $80 \mathrm{~mL}$ of contrast material - first $50 \mathrm{~mL}$ of contrast material, followed by $30 \mathrm{~mL}$ contrast material diluted with $30 \mathrm{~mL}$ normal saline, followed by $50 \mathrm{~mL}$ of normal saline - all at $5 \mathrm{~mL} / \mathrm{s}$. All examinations were performed using retrospective gating with tube current modulation ( $100 \%$ peak tube current during the mid-end-diastole and up to $80 \%$ reduction at end systole) to reduce radiation exposure.

\section{CT image reconstruction and postprocessing}

ECG-gated images were retrospectively reconstructed at $1.25-\mathrm{mm}$ slice thickness at $1.25-\mathrm{mm}$ intervals at $5 \%$ increments from $0-95 \%$ of the R-R interval of the ECG, for a total of 20 phases at the CT scanner console (Cardiac Volume Navigator, GE Healthcare). All reconstructed images were sent to and postprocessed on a GE workstation (Advantage Windows Workstation version 4.5, GE Healthcare) using the Automated Ejection Fraction protocol in the CardIQ function software (GE Healthcare, Wisconsin). After launching the Auto Ejection Fraction protocol, the LV/RV volumes and ejection fractions are automatically calculated and displayed for all selected phases loaded. The edit valve button is only available for the LV, which allows repositioning relative to the mitral valve plane (Fig. 2), which recalculates cavity segmentation. The short-axis and horizontal and vertical long-axis views were obtained with autosegmentation of both the right and left ventricles (Figs 3,4 ). The automatically obtained endocardial contours were visually reviewed in all planes (short axis, horizontal and vertical long axes) for correctness to ensure inclusion of ventricular outflow tracts (just distal to the aortic and pulmonary valves) and cardiac apex throughout each of the 20 cardiac phases reconstructed (Figs. 1-3) and, if necessary, manually edited/adjusted in a meticulous fashion using valve edit, add and remove structure tools. Papillary muscles and left ventricular outflow tracts were included in the LV cavity. Trabeculations and right ventricular outflow tracts were included in the RV cavity. The analysis software provided calculation of (i) LV and RV volume including enddiastolic volume (EDV) and end-systolic volume (ESV); (ii) LV and RV stroke volume (SV); (iii) LV and RV ejection function (EF); and (iv) left ventricular myocardial mass. The following measurement definitions were used:

- EDV: maximal ventricular volume when the cavity is largest

- ESV: minimal ventricular volume when the ventricular cavity is smallest

- SV: the difference between EDV and ESV

- EF: the fraction of EDV ejected with each heart beat (SV/EDV times 100).

- Indexed values: normalised to BSA

Volumetric and functional analysis was performed independently on all examinations by a fellowshiptrained cardiothoracic radiologist with 4 years of experience and a cardiovascular imaging fellowship-trained cardiologist with 2 years of experience, each blinded to patient information.

The LV and RV volume and function analysis using the automated software takes approximately $5 \mathrm{~min}$ if the optimal threshold of the luminal blood pool is obtained and approximately $10 \mathrm{~min}$ if no optimal threshold of the luminal blood pool (usually the RV) is obtained, in which case more extensive user interaction is required. 

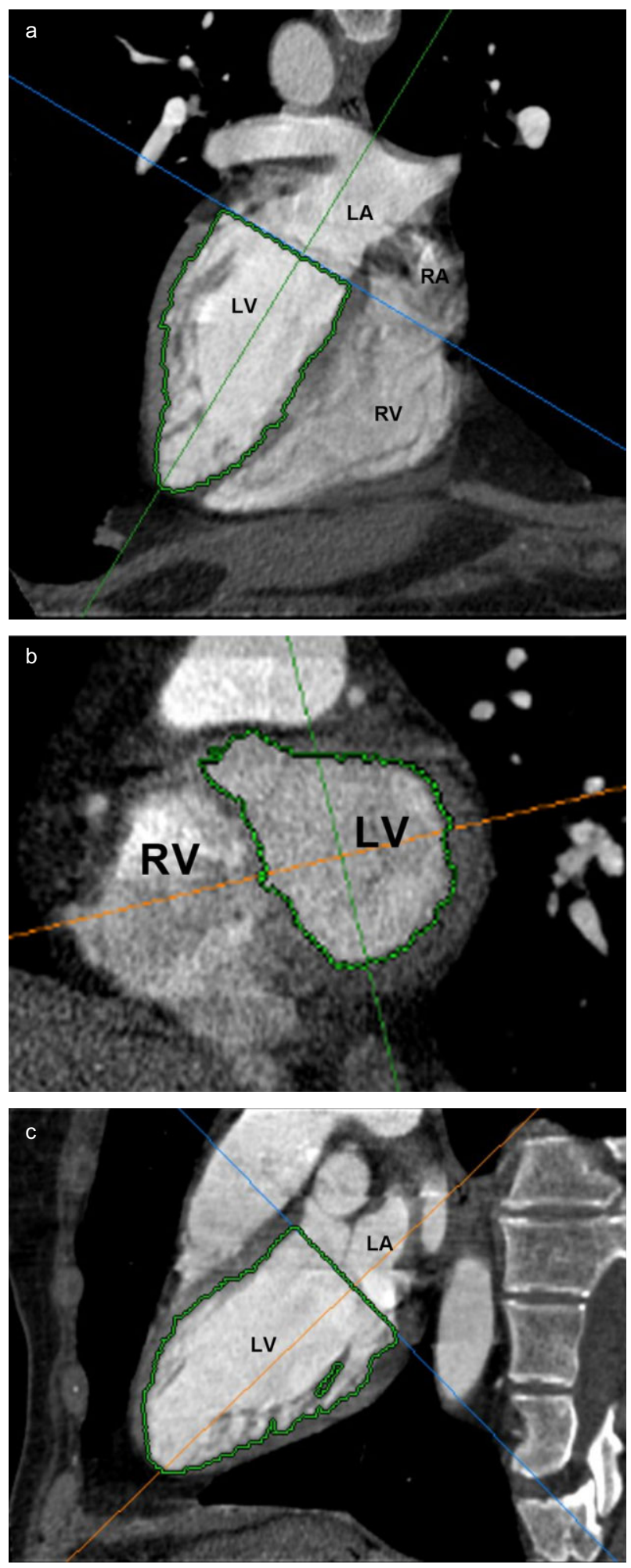

Fig. 2. Views of the left and right ventricles demonstrating adjustment of long and short planes using valve edit tool. (a) Horizontal long-axis view. (b) Shortaxis view. (c) Vertical long-axis view. LA, left atrium; LV, left ventricle; RA, right atrium; RV, right ventricle.
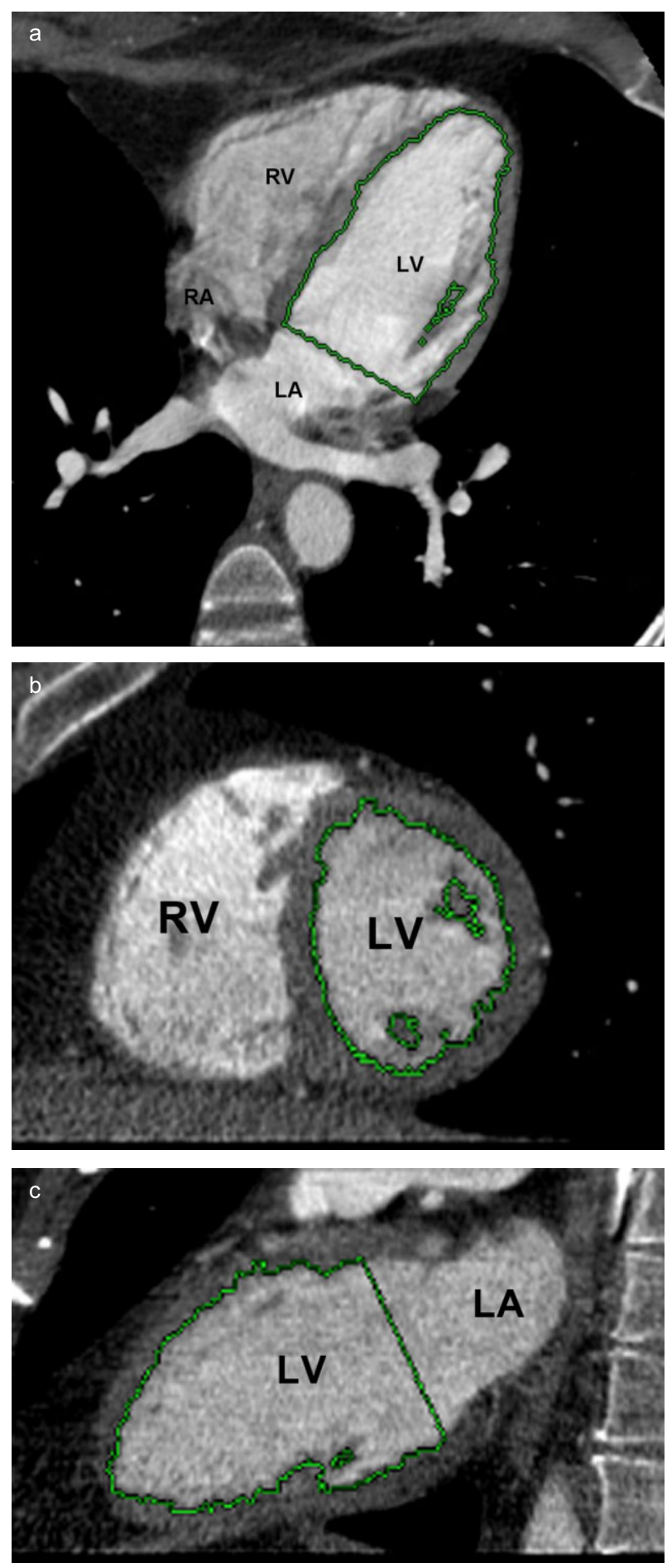

Fig. 3. Endocardial tracings of the left ventricle. (a) Horizontal long-axis view (b) Short-axis view, involving left ventricular outflow tract; papillary muscles were also included in LV mass analysis. (c) Vertical long-axis view. LA, left atrium; LV, left ventricle; RA, right atrium; RV, right ventricle. 

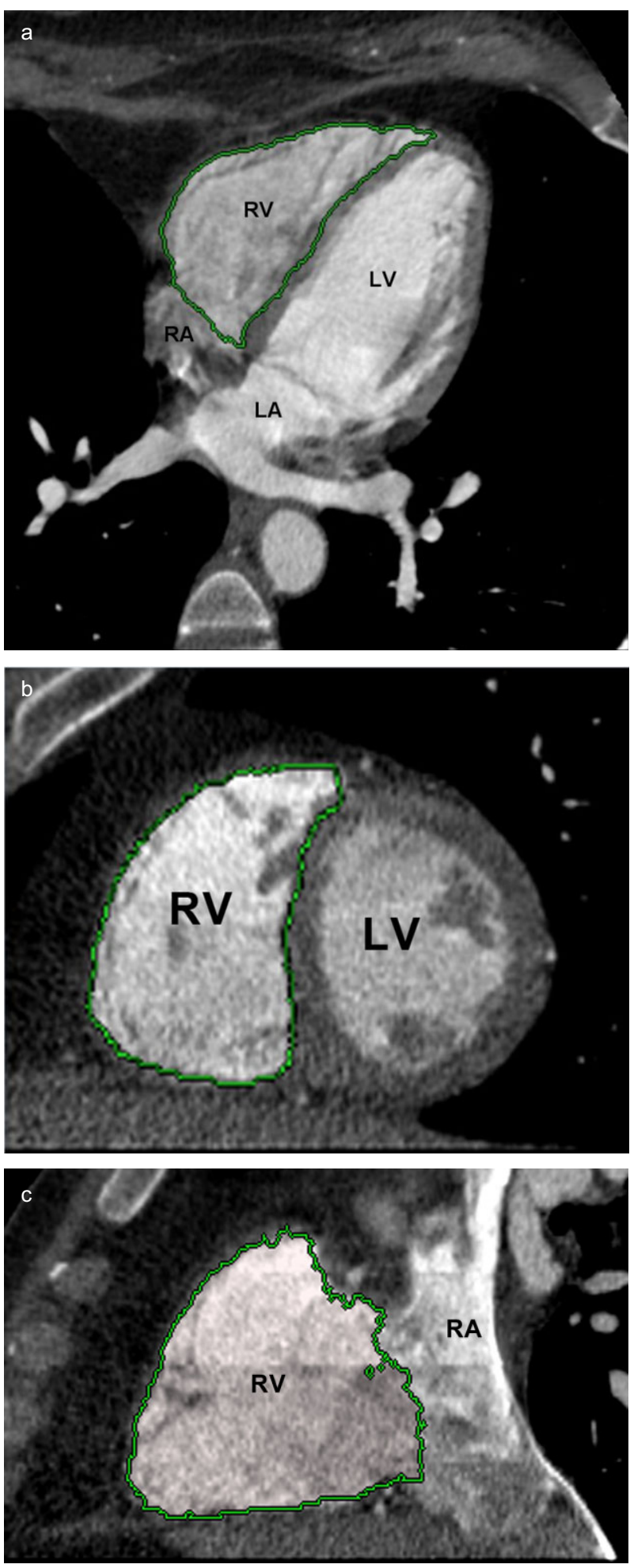

Fig. 4. Endocardial tracings of the right ventricle. (a) Horizontal long-axis view. (b) Short-axis view. (c) Vertical long-axis view. LA, left atrium; LV, left ventricle; $\mathrm{RA}$, right atrium; RV, right ventricle.

\section{Hounsfield unit measurements}

The Automated Ejection Fraction protocol is a thresholdbased region-growing segmentation approach that usually requires minimal to almost no user interaction if the optimal threshold is reached. For adequate segmentation of the left and right ventricles, a minimum of 150 Hounsfield units ( $\mathrm{HU}$ ) of contrast enhancement of the ventricular cavity is needed. To test for optimal contrast opacification and automatic segmentation, mean $\mathrm{HU}$ measurements were obtained in 72 subjects using the region of interest tool of the $L V$ and $R V$ blood pool.

\section{Reproducibility}

To test for inter-reader variability of contouring, the data from 69 randomly selected subjects were independently analysed by a second reader 1 year later, using the same methodology and blinded to patient information and previous calculations.

\section{Definitions}

BMI was defined as the individual's body mass $(\mathrm{kg})$ divided by the square of his/her height $\left(\mathrm{m}^{2}\right)$, with a normal BMI range of $18.5-25 \mathrm{~kg} / \mathrm{m}^{2}$. Individuals with BMI less than 18.5 are defined as underweight, individuals with BMI between $25-30 \mathrm{~kg} / \mathrm{m} 2$ as overweight and individuals with BMI above $30 \mathrm{~kg} / \mathrm{m} 2$ as obese.

\section{Statistical analysis}

Continuous data are presented as mean \pm standard deviation or median (interquartile range) where appropriate. Categorical data are presented as numbers and percentages. The univariate association between the tested variables was calculated with Student's $t$-test for continuous variables with normal distribution, MannWhitney $U$-test for ordinal variables or continuous variables without normal distribution, and $\chi^{2}$ or Fisher's exact test for categorical variables as appropriate. The data were summarised using descriptive statistics. To test the independent univariate and multivariate associations and statistical significance of LV and RV volume and function values between the age groups after adjusting for gender, BSA and age as a continuous variable, a general linear model analysis was performed. A method comparison test or difference of means test as described by Bland and Altman ${ }^{14}$ was used to assess inter-reader agreement. A $P$-value of $<0.05$ was considered statistically significant. All computations were performed with SAS/STAT (Version 9.2, SAS Institute Inc, Cary, NC, USA).

\section{Results}

\section{Baseline characteristics}

The study population of 74 patients $(38 / 74,51 \%$ men and $36 / 74,49 \%$ female) consisted of $60 / 74(81 \%)$ 
Caucasians, 10/74 (14\%) African Americans, 3/74 (4\%) Asians and one (1\%) Hispanic. The patients ranged in age from 30 to 70 years (mean age $49.9 \pm 11.1$ years) (Table 1). The subjects were stratified into four age groups based on age in decades. The mean heart rate was $73 \pm 12 \mathrm{bpm}$ before the scan and was $56.9 \pm 0.2 \mathrm{bpm}$ during the scan. The ventricular functional parameters for males and females stratified by age groups for each ventricle are presented in Tables 2-5, and ventricular functional parameters for all subjects and stratified by gender for each ventricle are presented in Tables 6 and 7.

\section{Influence of BMI}

Our study population contained $22 / 73$ (30\%) normal, $29 / 73(40 \%)$ overweight and $22 / 73$ (30\%) obese subjects. There was a statistically significant difference in the BMI distribution according to BMI group ( $P$-value of $<0.0001)$. There was no statistically significant difference in the BMI distribution according to gender ( $P$-value of 0.6 , Table 1). A statistically significant difference in the
Table 1. Baseline characteristics of normal subjects

\begin{tabular}{|c|c|c|c|c|}
\hline Age (years) & $30-39$ & $40-49$ & $50-59$ & $60-70$ \\
\hline \multicolumn{5}{|l|}{ Females } \\
\hline$N$ & 8 & 10 & 9 & 9 \\
\hline Weight (kg) & $87 \pm 22$ & $74 \pm 14$ & $75 \pm 9$ & $68 \pm 10$ \\
\hline Height (cm) & $165 \pm 10$ & $165 \pm 13$ & $163 \pm 5$ & $64 \pm 5$ \\
\hline $\mathrm{BMI}\left(\mathrm{kg} / \mathrm{m}^{2}\right)$ & $32 \pm 6$ & $33 \pm 7$ & $37 \pm 9$ & $42 \pm 9$ \\
\hline $\mathrm{BSA}\left(\mathrm{m}^{2}\right)$ & $2 \pm 0.3$ & $1.8 \pm 0.2$ & $1.8 \pm 0.1$ & $1.7 \pm 0.1$ \\
\hline $\mathrm{SBP}(\mathrm{mmHg})$ & $122 \pm 8$ & $123 \pm 10$ & $126 \pm 9$ & $120 \pm 9$ \\
\hline $\mathrm{DBP}(\mathrm{mmHg})$ & $74 \pm 7$ & $77 \pm 12$ & $77 \pm 8$ & $65 \pm 8$ \\
\hline \multicolumn{5}{|l|}{ Males } \\
\hline$N$ & 10 & 10 & 10 & 8 \\
\hline Weight (kg) & $90 \pm 13$ & $86 \pm 15$ & $91 \pm 22$ & $90 \pm 15$ \\
\hline Height (cm) & $180 \pm 5$ & $175 \pm 10$ & $183 \pm 8$ & $180 \pm 5$ \\
\hline BMI $\left(\mathrm{kg} / \mathrm{m}^{2}\right)$ & $28 \pm 3$ & $43 \pm 15$ & $41 \pm 11$ & $41 \pm 14$ \\
\hline $\operatorname{BSA}\left(m^{2}\right)$ & $2 \pm 0.2$ & $2 \pm 0.2$ & $2.1 \pm 0.3$ & $2.1 \pm 0.2$ \\
\hline SBP (mmHg) & $120 \pm 7$ & $123 \pm 12$ & $123 \pm 11$ & $124 \pm 11$ \\
\hline $\mathrm{DBP}(\mathrm{mmHg})$ & $70 \pm 7$ & $77 \pm 8$ & $74 \pm 11$ & $76 \pm 8$ \\
\hline
\end{tabular}

All data are given as mean \pm SD unless otherwise indicated. BMI, body mass index; BSA, body surface area; DBP, Diastolic blood pressure; SBP, systolic blood pressure.

Table 2. Female left ventricular function and volume by age

\begin{tabular}{lcccc}
\hline Age (years) & $30-39$ & $40-49$ & $50-59$ & $60-70$ \\
\hline Absolute values & & & & \\
EDV $(\mathrm{mL})$ & $146(121-171)$ & $142(127-157)$ & $143(131-154)$ & $150(118-182)$ \\
ESV $(\mathrm{mL})$ & $40(30-50)$ & $43(36-50)$ & $49(34-64)$ & $42(36-48)$ \\
SV $(\mathrm{mL})$ & $106(88-123)$ & $99(88-110)$ & $94(86-102)$ & $109(80-137)$ \\
EF $(\%)$ & $73(69-77)$ & $70(67-73)$ & $67(59-75)$ & $72(68-76)$ \\
Mass $(\mathrm{g})$ & $76(57-95)$ & $76(64-90)$ & $77(66-88)$ & $73(67-79)$ \\
Indexed values & & & & $87(63-111)$ \\
EDV/BSA $\left(\mathrm{mL} / \mathrm{m}^{2}\right)$ & $75(62-88)$ & $77(70-84)$ & $27(18-35)$ & $24(20-28)$ \\
ESV/BSA $\left(\mathrm{mL} / \mathrm{m}^{2}\right)$ & $21(15-27)$ & $23(20-26)$ & $51(46-56)$ & $63(42-84)$ \\
SV/BSA $\left(\mathrm{mL} / \mathrm{m}^{2}\right)$ & $54(46-61)$ & $54(48-60)$ & $42(35-49)$ & $42(37-46)$ \\
Mass $\left(\mathrm{g} / \mathrm{m}^{2}\right)$ & $38(30-46)$ & $42(37-47)$ & & \\
\hline
\end{tabular}

All values given as mean ( $95 \%$ confidence interval). BSA, body surface area; EDV, end-diastolic volume; EF, ejection fraction; ESV, end-systolic volume; SV, stroke volume.

Table 3. Male left ventricular function and volume by age

\begin{tabular}{|c|c|c|c|c|}
\hline Age (years) & $30-39$ & $40-49$ & $50-59$ & $60-70$ \\
\hline \multicolumn{5}{|l|}{ Absolute values } \\
\hline EDV $(\mathrm{mL})$ & 165 (159-171) & 157 (137-177) & $152(139-165)$ & $151(129-172)$ \\
\hline ESV (mL) & 55 (48-62) & $46(35-57)$ & $43(35-51)$ & $45(28-62)$ \\
\hline SV (mL) & $110(106-114)$ & $111(94-127)$ & $109(98-120)$ & $106(93-119)$ \\
\hline $\mathrm{EF}(\%)$ & $67(63-70)$ & 65 (49-81) & $72(68-76)$ & $71(62-79)$ \\
\hline Mass (g) & $110(104-116)$ & $109(92-126)$ & $118(101-135)$ & $113(101-125)$ \\
\hline \multicolumn{5}{|l|}{ Indexed values } \\
\hline EDV/BSA $\left(\mathrm{mL} / \mathrm{m}^{2}\right)$ & $78(74-81)$ & 77 (66-88) & $73(66-80)$ & $73(63-73)$ \\
\hline $\mathrm{ESV} / \mathrm{BSA}\left(\mathrm{mL} / \mathrm{m}_{2}\right)$ & $26(23-29)$ & $23(18-28)$ & $21(17-25)$ & $22(13-30)$ \\
\hline SV/BSA $\left(\mathrm{mL} / \mathrm{m}^{2}\right)$ & $52(48-55)$ & $55(46-64)$ & $52(48-56)$ & $51(46-56)$ \\
\hline Mass $\left(\mathrm{g} / \mathrm{m}^{2}\right)$ & $52(47-57)$ & $53(45-61)$ & $55(50-60)$ & $55(47-63)$ \\
\hline
\end{tabular}

All values given as mean (95\% confidence interval). BSA, body surface area; EDV, end-diastolic volume; EF, ejection fraction; ESV, end-systolic volume; SV, stroke volume. 
Table 4. Female right ventricular function and volume by age

\begin{tabular}{lcccc}
\hline Age (years) & $30-39$ & $40-49$ & $50-59$ & $60-70$ \\
\hline Absolute values & & & & \\
EDV $(\mathrm{mL})$ & $173(146-200)$ & $157(138-176)$ & $169(150-187)$ & $155(133-177)$ \\
ESV $(\mathrm{mL})$ & $74(56-92)$ & $56(32-80)$ & $78(57-99)$ & $57(36-78)$ \\
SV $(\mathrm{mL})$ & $99(83-115)$ & $101(87-115)$ & $91(80-102)$ & $97(82-112)$ \\
EF $(\%)$ & $58(51-65)$ & $66(53-79)$ & $54(46-62)$ & $64(52-76)$ \\
Indexed values & & & & \\
EDV/BSA $\left(\mathrm{mL} / \mathrm{m}^{2}\right)$ & $88(75-100)$ & $85(78-92)$ & $92(85-99)$ & $88(78-97)$ \\
ESV/BSA $\left(\mathrm{mL} / \mathrm{m}^{2}\right)$ & $38(28-48)$ & $30(18-42)$ & $42(33-51)$ & $32(20-44)$ \\
SV/BSA $\left(\mathrm{mL} / \mathrm{m}^{2}\right)$ & $50(45-55)$ & $55(47-63)$ & $50(44-56)$ & $56(47-64)$ \\
\hline
\end{tabular}

All values given as mean ( $95 \%$ confidence interval). BSA, body surface area; EDV, end-diastolic volume; EF, ejection fraction; ESV, end-systolic volume; SV, stroke volume.

Table 5. Male right ventricular function and volume by age

\begin{tabular}{lccrr}
\hline Age (years) & $30-39$ & $40-49$ & $50-59$ & $60-70$ \\
\hline Absolute values & & & & \\
$\quad$ EDV $(\mathrm{mL})$ & $214(197-230)$ & $197(169-225)$ & $182(160-204)$ & $203(74-131)$ \\
ESV $(\mathrm{mL})$ & $100(71-128)$ & $84(52-115)$ & $71(47-95)$ & $91(58-124)$ \\
SV $(\mathrm{mL})$ & $113(96-130)$ & $113(91-135)$ & $111(98-124)$ & $112(97-126)$ \\
EF (\%) & $54(42-66)$ & $59(45-72)$ & $62(51-73)$ & $57(45-67)$ \\
Indexed values & $101(94-107)$ & $96(84-107)$ & $86(77-95)$ & $99(84-114)$ \\
EDV/BSA (mL/m²) & $47(34-60)$ & $41(26-56)$ & $34(22-45)$ & $45(27-63)$ \\
ESV/BSA $\left(\mathrm{mL} / \mathrm{m}^{2}\right)$ & $54(43-65)$ & $56(44-67)$ & $52(45-58)$ & $54(47-61)$ \\
$\quad$ SV/BSA $\left(\mathrm{mL} / \mathrm{m}^{2}\right)$ & & & & \\
\hline
\end{tabular}

All values given as mean (95\% confidence interval). BSA, body surface area; EDV, end-diastolic volume; EF, ejection fraction; ESV, end-systolic volume; SV, stroke volume.

distribution of normal and overweight subjects was seen only for RV EF with mean of $67 \pm 20$ and $56 \pm 10$ ( $P$-value of 0.005$)$, absolute RV EDV with mean of $167 \pm 35$ and $189 \pm 34(P$-value of 0.001$)$ and absolute RV ESV with mean of $59 \pm 44$ and $85 \pm 28$ ( $P$-value of 0.003). After adjustment for age groups and gender, the only statistically significant differences of BMI were in RV EF ( $P$-value of 0.01$)$ and absolute RV ESV $(P$-value of 0.03$)$.

\section{Influence of gender}

Mean \pm SD with $95 \%$ confidence intervals for men and women are presented in Table 3. Men had statistically

Table 6. Left ventricular volume and function for all ages

\begin{tabular}{lccc}
\hline & All subjects $(n=74)$ & Females $(n=36)$ & Males $(n=38)$ \\
\hline Absolute values & & & \\
$\quad$ EDV $(\mathrm{mL})$ & $151 \pm 25(145-157)$ & $145 \pm 28(136-154)$ & $157 \pm 22(150-164)$ \\
ESV $(\mathrm{mL})$ & $45 \pm 14(42-48)$ & $43 \pm 13(39-47)$ & $47 \pm 15(42-52)$ \\
SV $(\mathrm{mL})$ & $106 \pm 20(101-110)$ & $102 \pm 23(94-109)$ & $109 \pm 16(104-114)$ \\
EF $(\%)$ & $69 \pm 10(67-71)$ & $70 \pm 7(68-72)$ & $69 \pm 13(65-73)$ \\
Mass $(g)$ & $94 \pm 25(88-100)$ & $76 \pm 16(71-81)$ & $112 \pm 19(106-118)$ \\
Indexed values & $77 \pm 15(74-80)$ & $79 \pm 18(73-85)$ & $76 \pm 11(72-79)$ \\
EDV/BSA (mL/m²) & $23 \pm 7(21-25)$ & $24 \pm 7(22-26)$ & $23 \pm 7(21-25)$ \\
ESV/BSA $\left(\mathrm{mL} / \mathrm{m}^{2}\right)$ & $54 \pm 12(51-57)$ & $56 \pm 15(51-61)$ & $53 \pm 8(50-55)$ \\
SV/BSA $\left(\mathrm{mL} / \mathrm{m}^{2}\right)$ & $47 \pm 10(45-49)$ & $41 \pm 8(38-44)$ & $54 \pm 9(51-57)$ \\
Mass/BSA $\left(\mathrm{g} / \mathrm{m}^{2}\right)$ & & & \\
\hline
\end{tabular}

All data given as mean \pm SD $(95 \%$ confidence interval. BSA, body surface area; EDV, end-diastolic volume; EF, ejection fraction; ESV, end-systolic volume; SV, stroke volume. 
Table 7. Right ventricular volume and function for all ages

\begin{tabular}{lccc}
\hline & All subjects $(n=74)$ & Females $(n=36)$ & Males $(n=38)$ \\
\hline Absolute values & & & \\
$\quad$ EDV $(\mathrm{mL})$ & $181 \pm 36(174-188)$ & $163 \pm 28(155-171)$ & $199 \pm 34(190-208)$ \\
ESV $(\mathrm{mL})$ & $76 \pm 36(69-83)$ & $66 \pm 28(58-74)$ & $86 \pm 40(75-97)$ \\
SV $(\mathrm{mL})$ & $105 \pm 22(101-109)$ & $97 \pm 18(92-102)$ & $112 \pm 23(106-118)$ \\
EF (\%) & $59 \pm 15(56-62)$ & $61 \pm 14(51-65)$ & $58 \pm 16(54-62)$ \\
Indexed values & & & \\
EDV/BSA $\left(\mathrm{mL} / \mathrm{m}^{2}\right)$ & $92 \pm 14(89-95)$ & $88 \pm 12(85-91)$ & $96 \pm 15(92-100)$ \\
ESV/BSA $\left(\mathrm{mL} / \mathrm{m}^{2}\right)$ & $39 \pm 17(56-42)$ & $36 \pm 15(32-40)$ & $42 \pm 19(37-47)$ \\
SV/BSA $\left(\mathrm{mL} / \mathrm{m}^{2}\right)$ & $53 \pm 11(51-55)$ & $53 \pm 10(50-56)$ & $54 \pm 13(50-57)$ \\
\hline
\end{tabular}

All data given as mean \pm SD $(95 \%$ confidence interval. BSA, body surface area; EDV, end-diastolic volume; EF, ejection fraction; ESV, end-systolic volume; SV, stroke volume.

significantly higher absolute and indexed RV EDV ( $P$-value of $<0.0001$ for absolute and $P$-value of 0.006 for indexed values), absolute RV ESV ( $P$-value of 0.008 ) and absolute RV SV ( $P$-value of 0.001$)$ as well as higher absolute LV EDV ( $P$-value of 0.04$)$. Men also had statistically significantly higher absolute and indexed LV mass values ( $P$-values of $<0.0001)$. A significant independent influence of gender on absolute RV EDV, RV ESV and LV mass (absolute and indexed), even after adjusting for age and BSA, was demonstrated. Otherwise, the genders had no significant differences, particularly in ejection fraction ( $P$-value of 0.4 for LVEF and RVEF respectively).

\section{Influence of age}

The only statistically significant difference observed in LV and RV volume and function value distribution according to age group was found in women aged 50-59 years. Women aged 50-59 had a 7\% lower LVEF than women aged 30-39 ( $P=0.03)$, with an expected mean LVEF for females of $67 \%$ versus $74 \%$, respectively. Similarly, men aged 50-59 had $31 \mathrm{~mL}$ lower absolute and $15 \mathrm{~mL}$ lower indexed RV EDV than men aged 30-39 $(P=0.027)$.

\section{Influence of BSA}

On multivariable analysis, BSA was found to have significant independent influence on absolute LV mass $(P=0.01)$, RV EDV $(P=0.03)$ and $\operatorname{RV} \operatorname{SV}(P=0.04)$ values.

\section{Hounsfield unit measurements}

The mean HU measurements of LV and RV blood pools were $346 \pm 74$ and $270 \pm 106$ respectively. Only four of 72 subjects $(0.05 \%)$ had RV blood pool mean $\mathrm{HU}$ of less than 150, a lower-bound threshold for optimal autosegmentation of the ventricular cavity. In these cases, the endocardial RV contours had to be corrected manually. No statistically significant difference was obtained between the LV and RV blood pool mean HU measurements.

\section{Inter-reader agreement}

There was no statistically significant difference in ventricular volume and functional parameters between readers by either Pearson's correlation coefficient or Bland-Altman analysis (Tables 8,9; Figs 5,6).

Table 8. Inter-rater variability between two raters for left ventricular volume, function and mass

\begin{tabular}{|c|c|c|c|c|c|c|c|c|}
\hline & \multicolumn{4}{|c|}{ Pearson correlation of the means of two raters $(n=69)$} & \multicolumn{4}{|c|}{ Method comparison test using Bland-Altman procedure $(n=69)$} \\
\hline & $\begin{array}{c}\text { Mean, rater } 1 / \\
\text { rater } 2\end{array}$ & $\begin{array}{l}\text { SD, rater } 1 / \\
\text { rater } 2\end{array}$ & Pearson's $r$ & $P$-value & $\begin{array}{c}\text { Mean difference } \\
\text { of two raters }\end{array}$ & $\begin{array}{c}\text { SD of the mean } \\
\text { difference of } \\
\text { two raters }\end{array}$ & Pearson's $r$ & $P$-value \\
\hline $\mathrm{EDV}(\mathrm{mL})$ & $151 / 146$ & $25 / 25$ & 0.65 & $<0.001$ & 2.57 & 10.74 & 0.04 & 0.71 \\
\hline ESV $(m L)$ & $45 / 41$ & $14 / 13$ & 0.77 & $<0.001$ & 1.99 & 4.64 & 0.05 & 0.65 \\
\hline SV (mL) & $106 / 105$ & $20 / 20$ & 0.63 & $<0.001$ & 0.38 & 8.68 & 0.00 & 0.99 \\
\hline EF (\%) & 70/72 & $7 / 8$ & 0.74 & $<0.001$ & -0.90 & 2.62 & -0.11 & 0.37 \\
\hline Mass (g) & $95 / 89$ & $25 / 25$ & 0.93 & $<0.001$ & 2.41 & 4.69 & -0.06 & 0.63 \\
\hline
\end{tabular}

All data given as mean \pm SD (95\% confidence interval. EDV, end-diastolic volume; EF, ejection fraction; ESV, end-systolic volume; SV, stroke volume. 
Table 9 Inter-rater variability between two raters for right ventricular volume and function

\begin{tabular}{|c|c|c|c|c|c|c|c|c|}
\hline & \multicolumn{4}{|c|}{ Pearson correlation of the means of two raters $(n=69)$} & \multicolumn{4}{|c|}{ Method comparison test using Bland-Altman procedure $(n=69)$} \\
\hline & $\begin{array}{c}\text { Mean, rater } 1 / \\
\text { rater } 2\end{array}$ & $\begin{array}{l}\text { SD, rater } 1 / \\
\text { rater } 2\end{array}$ & Pearson's $r$ & $P$-value & $\begin{array}{c}\text { Mean difference } \\
\text { of two raters }\end{array}$ & $\begin{array}{c}\text { SD of the mean } \\
\text { difference of } \\
\text { two raters }\end{array}$ & Pearson's $r$ & $P$-value \\
\hline $\mathrm{EDV}(\mathrm{mL})$ & 182/177 & $35 / 33$ & 0.91 & $<0.001$ & 3.12 & 7.1 & 0.11 & 0.36 \\
\hline ESV (mL) & 77/75 & $35 / 33$ & 0.92 & $<0.001$ & 1.68 & 6.8 & 0.06 & 0.63 \\
\hline SV (mL) & $105 / 102$ & $22 / 24$ & 0.92 & $<0.001$ & 1.17 & 4.5 & -0.02 & 0.053 \\
\hline EF (\%) & $59 / 58$ & $14 / 14$ & 0.93 & $<0.001$ & -0.01 & 2.6 & -0.03 & 0.78 \\
\hline
\end{tabular}

All data given as mean \pm SD (95\% confidence interval. EDV, end-diastolic volume; EF, ejection fraction; ESV, end-systolic volume; SV, stroke volume.

\section{Discussion}

We established mean values and $95 \%$ confidence interval for LV and RV volumes, function and LV mass by MDCT in healthy patients without hypertension and hypercholesterolaemia, stratified by age, gender and BSA. Our results demonstrate significant gender differences for LV mass and ventricular volumes.

We believe that these data have significant impact for current and future clinical practice and research. Previously published MDCT data have compared favorably with $\mathrm{CMR}^{15}$ which is considered the current gold standard for LV and RV volume and function.

Of note, significant differences can exist among measurements obtained with different software tools (semiautomated versus automated) from the same dataset. ${ }^{15}$ Many previously published studies used semi-automated methods with Simpson's method to assess LV and RV volume and function without inclusion of the outflow tracts in analysis.8,9 Our absolute volumetric values were slightly higher, especially in women, when compared with the values reported by Maceira et al. using CMR; however, the volumetric values indexed to BSA are similar. ${ }^{8,9}$ These authors had not included the outflow tracts in their chamber analysis, which likely accounts for the difference. Furthermore, our absolute volumetric measurements are slightly higher, but our indexed values are similar to prior observations with MDCT using 3D measures using the Hounsfield unit-based endocardial border detection technique with manual correction, which is an older version (4.3) of our currently used software (4.5). ${ }^{16}$

\section{Influence of age and gender on LV and RV volume, function and mass}

Age alone may not have a significant impact on ventricular function and mass, ${ }^{17,18}$ but age in concert with gender demonstrated a significant difference in volume in men and function in women. These observations parallel autopsy findings with progressive myocyte loss that occurs with increasing age in men and values remaining constant in women. ${ }^{19}$ Such age-related gender differ- ences may result from a reduction of physical activity with age and also from reduced testosterone level with age in men, which may explain the reduced ventricular mass. ${ }^{18}$ Also, we observed that myocardial hypertrophy does not develop with age in either women or men, consistent with previously published autopsy findings. ${ }^{19}$ Similar to prior reports, gender independently had a significant influence on LV mass and ventricular volumes. . $^{8,9,17,18}$

\section{Effect of BSA on LV and RV volume, function and mass}

As expected, BSA also had a significant impact on LV mass and RV volumes. This indicates the need for and the appropriateness of indexing LV/RV volume and LV mass to BSA when reporting these parameters.

To our knowledge, our study is the first to report age-, gender- and BSA-specific reference ranges of LV and RV volume, function and mass with MDCT using thresholdbased function analysis software.

\section{Reproducibility}

We demonstrated excellent reproducibility of LV functional parameters and RV functional parameters. We could have expected differences in RV volume and function, mainly explained by the difficulty in defining the most basal slice. especially in the cases where the endocardial RV contours had to be corrected manually secondary to lower-bound threshold for optimal autosegmentation of the RV. Prior studies demonstrated higher variability of RV measurements than for the LV, further illustrating the complexity of RV measurements. ${ }^{18}$

\section{Clinical application}

The increasing availability and use of contrast-enhanced retrospectively ECG-gated CT in the care of cardiac patients allows volumetric and functional analysis of cardiac chambers. Impairment of LV and RV function is extremely important in patients with cardiomyopathy 
a
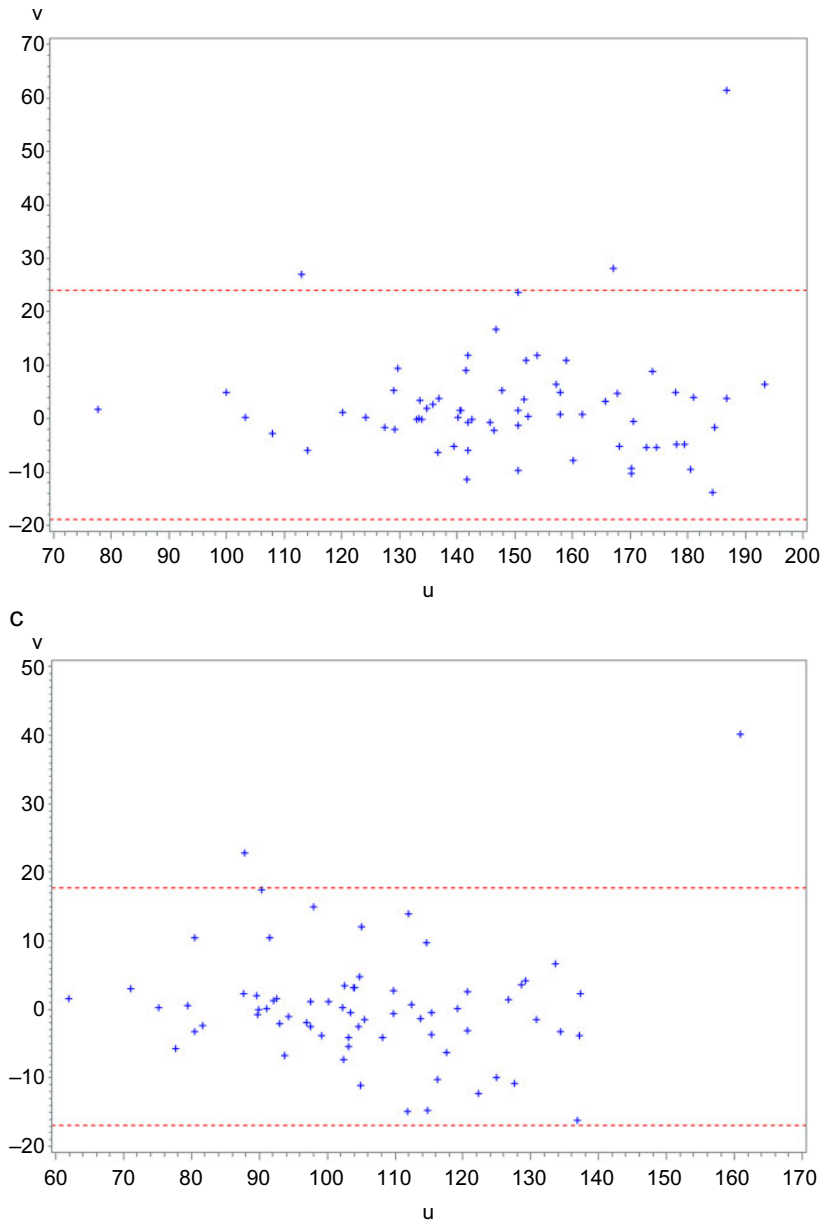

e

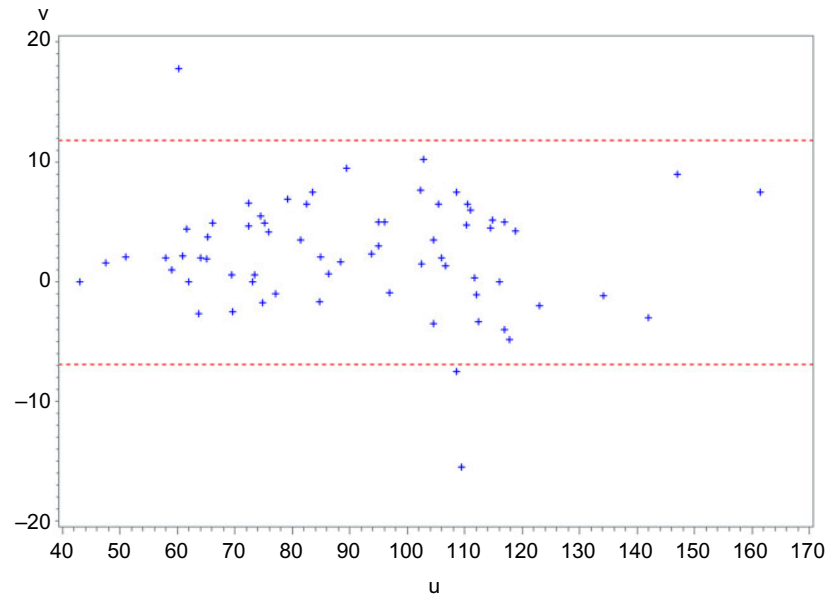

b
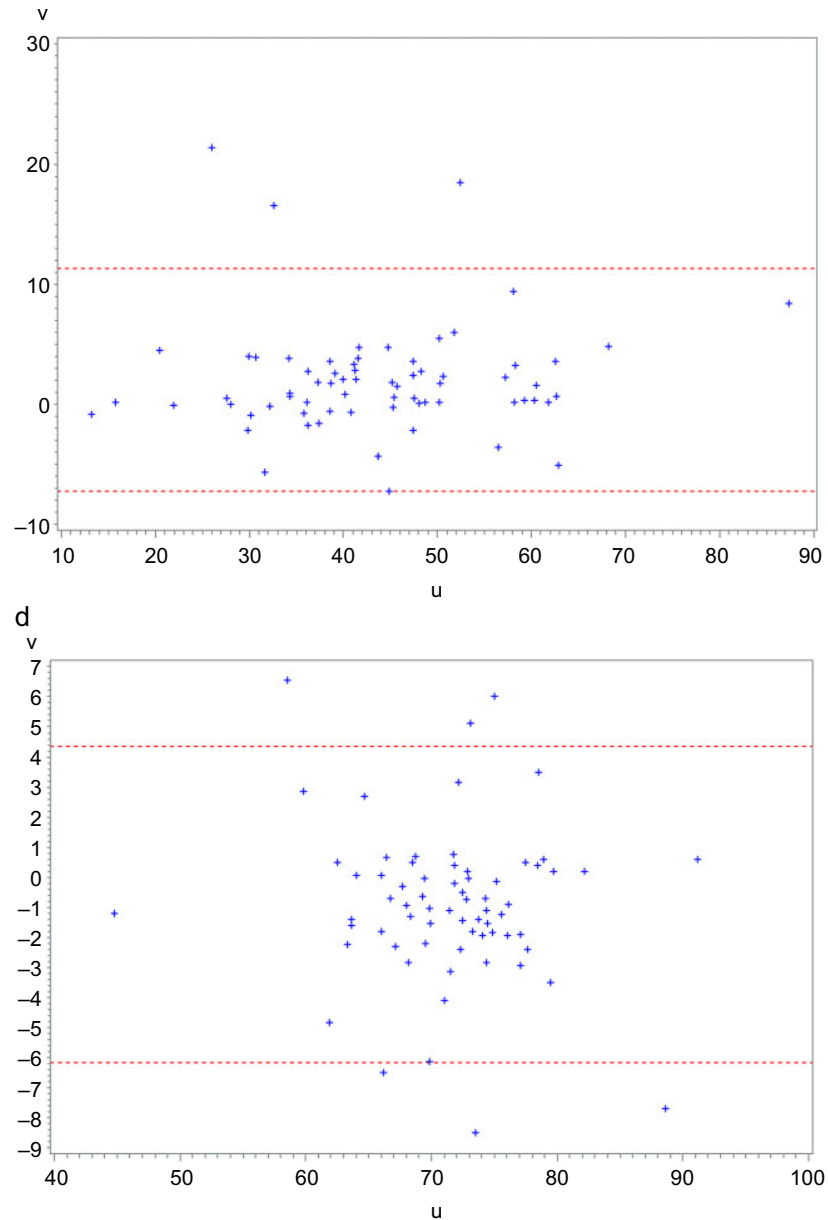

Fig. 5. Inter-reader agreement (Bland-Altman plots of mean differences and $95 \%$ confidence intervals for two readers) for left ventricular volume and function for 69 subjects. (a) End-diastolic volume. (b) End-systolic volume. (c) Stroke volume. (d) Ejection fraction. (e) Mass.

because of increased risk of sudden cardiac death. ${ }^{20}$ Even though MRI is an established gold standard for evaluation of ventricular function and volume, there is a relative contraindication of its use in patients with implantable cardioverter-defibrillator/pacemaker. Therefore, use of contrast-enhanced retrospectively ECG-gated CT as an alternative problem-solving tool in the care of cardiac patients and establishing reference 

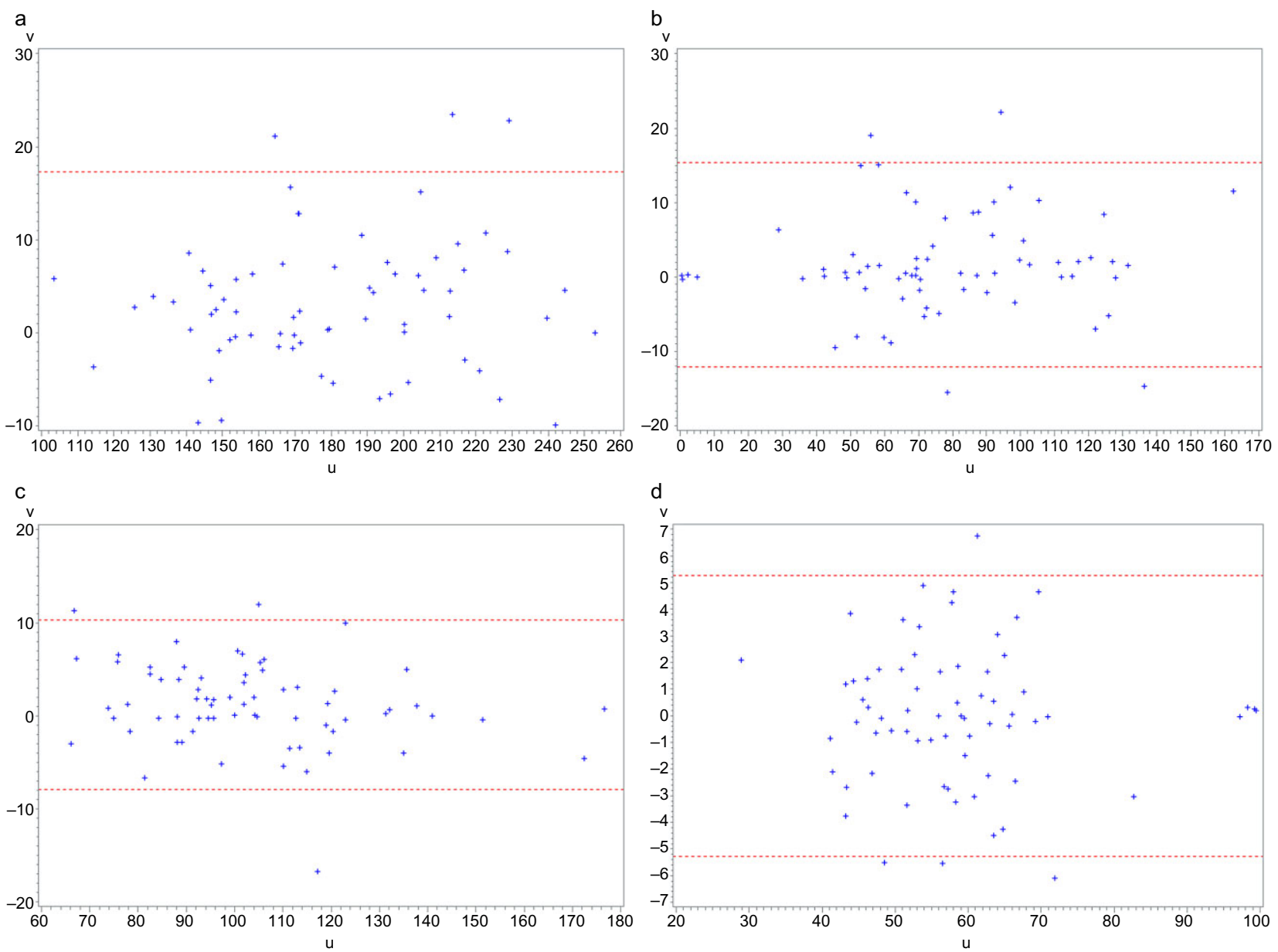

Fig. 6. Inter-reader agreement (Bland-Altman plots of mean differences and $95 \%$ confidence intervals for two readers) for right ventricular volume and function for 69 subjects. (a) End-diastolic volume. (b) End-systolic volume. (c) Stroke volume. (d) Ejection fraction.

normal ranges of LV and RV volume, function and diameter with MDCT are very important to risk-stratify patients with cardiac and cerebrovascular diseases.

\section{Limitations}

This is a retrospective study with a small sample size. There are radiation concerns that exist with MDCT, particularly when using the retrospective gating required for functional analysis, which likely relegates CT for functional analysis to either a problem-solving tool if echocardiography and MR are suboptimal and/or contraindicated or to incidental data reporting when retrospective gating has to be used. We attempted to exclude subjects with significant coronary artery disease, hypertension and hyperlipidaemia, so that our subjects represent overall healthy people free of cardiovascular disease (CVD).

Our study population included $30 \%$ obese subjects, a risk for metabolic syndrome and future cardiovascular disease; however, we stressed the importance of reporting normalised ventricular volumetric and functional values, which accounts for this limitation. As other functional assessments were not done at the same time, we could only compare our results with previously reported historical data. In the absence of a gold standard, it is not possible to estimate the accuracy of the described methodology; however, the ventricular functions served as internal controls for each other.

\section{Conclusion}

We have provided reference mean values of LV and RV volume, function and mass normalised by age, gender and body surface area for ECG-gated MDCT using threshold-based analysis software among subjects free of CVD. We stressed the importance of using reference values indexed to gender and BSA. These parameters should always be obtained and reported, especially given the availability of automated functional analysis tools 
that significantly decrease the postprocessing time, and add important information that can be used in the patient's clinical management.

\section{References}

1. Ogunyankin KO, Liu K, Lloyd-Jones DM, Colangelo LA, Gardin JM. Reference values of right ventricular end-diastolic area defined by ethnicity and gender in a young adult population: the CARDIA study. Echocardiography 2011; 28: 142-9.

2. Mor-Avi V, Sugeng L, Lindner JR. Imaging the forgotten chamber: is the devil in the boundary? Am Soc Echocardiog 2010; 23: 141-3.

3. Jiang L, Levine RA, Weyman AE. Echocardiographic assessment of right ventricular volume and function. Echocardiography 1997; 14: 189-205.

4. Nesser HJ, Tkalec W, Patel AR et al. Quantification of right ventricular volumes and ejection fraction by three-dimensional echocardiography in patients: comparison with magnetic resonance imaging and radionuclide ventriculography. Echocardiography 2006; 23: 666-80.

5. Kjaergaard J, Petersen CL, Kjaer A, Schaadt BK, Oh $\mathrm{JK}$, Hassager C. Evaluation of right ventricular volume and function by $2 \mathrm{D}$ and $3 \mathrm{D}$ echocardiography compared to MRI. Eur J Echocardiography 2006; 7: 430-8.

6. Grouthues F, Smith GC, Moon JC, Bellenger NG, Collins P, Klein HU, Pennell DJ. Comparison of interstudy reproducibility of cardiovascular magnetic resonance with two-dimensional echocardiography in normal subjects and in patients with heart failure or left ventricular hypertrophy. Am J Cardiol 2002; 90: 29-34.

7. Mahabadi AA, Samy B, Seneviratne SK, Toepker MH, Bamberg F, Hoffmann U, Truong QA. Quantitative assessment of left atrial volume by electrocardiographic-gated contrast-enhanced multidetector computed tomography. J Cardiovasc Comp Tomogr 2009; 3: 80-7.

8. Maceira AM, Prasad SK, Khan M, Pennell DJ. Normalized left ventricular systolic and diastolic function by steady state free precession cardiovascular magnetic resonance. J Cardiovasc Magn Res 2006; 8: 417-26.

9. Maceira AM, Prasad SK, Khan M, Pennell DJ. Reference right ventricular systolic and diastolic function normalized to age, gender and body surface area from steady-state free precession cardiovascular magnetic resonance. Eur Heart J 2006; 27: 2879-88.

10. Alfakih K, Thiele H, Plein S, Bainbridge GJ, Ridgway JP, Sivananthan MU. Comparison of right ventricular volume measurement between segmented k-space gradient-echo and steady-state free precession magnetic resonance imaging. J Magn Reson Imaging 2002; 16: 253-8.

11. Lin FY, Min JK. Assessment of cardiac volumes by multidetector computed tomography. J Cardiovasc Comp Tomogr 2008; 2: 258-62.

12. Wilson PW, D'Agostino R, Levy D, Belanger AM, Silbershatz $H$, Kannel WB. Prediction of coronary heart disease using risk factor categories. Circulation 1998; 97: 1837-47.

13. Mosteller RD. Simplified calculation of body surface area. N Engl J Med 1987; 317: 1098.

14. Bland JM, Altman DG. Statistical methods for assessing agreement between two methods of clinical measurement. Lancet 1986; 1: 307-10.

15. Alfakih K, Plein S, Thiele $H$, Jones $T$, Ridgway JP et al. Normal human left and right ventricular dimensions for MRI as assessed by turbo gradient echo and steady-state free precession imaging sequences. J Magn Reson Imaging 2003; 17: 323-9.

16. Van Ooijen PM, de Jonge GJ, Oudkerk M. Informatics in radiology: postprocessing pitfalls in using $\mathrm{CT}$ for automatic and semiautomatic determination of global left ventricular function. Radiographics 2012; 32: 589-99.

17. Lin FY, Devereux RB, Roman MJ et al. Cardiac chamber volumes, function, and mass as determined by 64-multidetector row computed tomography: mean values among healthy adults free of hypertension and obesity. JACC Cardiovasc Imaging 2008; 1: 782-6.

18. Hudsmith LE, Petersen SE, Francis JM et al. Normal human left and right ventricular and left atrial dimensions using steady state free precession magnetic resonance imaging. J Cardiovasc Magn Res 2005; 7: 775-82.

19. Olivetti G, Giordano G, Corradi D et al. Gender differences and aging: effects on the human heart. JACC 1995; 26: 1068-79.

20. Zipes DP et al. ACC/AHA/ESC 2006 guidelines for management of patients with ventricular arrhythmias and the prevention of sudden cardiac death. Circulation 2006; 114: e385-484. 